\title{
Intermediate and High-Mass Ion Beams from a 10-cm Duopigatron
}

\author{
P. D. Weber ${ }^{1}$ and R. M. Gilgenbach ${ }^{1}$ \\ Received December 7, 1983; revised February 22, 1984
}

\begin{abstract}
Experimental studies of a 10-cm Duopigatron as a source of argon, krypton, and xenon ion beams are reported. Source plasma instabilities are examined, and the mass dependence of oscillation frequencies and instability onset conditions are determined. Arc current and density oscillations are found to be associated with ion acoustic fluctuations with frequencies scaling as $1 / \mathrm{M}^{1 / 2}$. Langmuir probe measurements within the source plasma double layer are used to indicate the physical mechanism responsible for the observed large-amplitude arc current shifts. Ion beams have been extracted at energies up $1018 \mathrm{kV}$, and drain currents up to $540 \mathrm{~mA}$ for argon, $440 \mathrm{~mA}$ for krypton, and $520 \mathrm{~mA}$ for xenon have been achieved with source plasma densities in the range $10^{11}-10^{12} \mathrm{~cm}^{-3}$. Excellent agreement with existing theoretical models has been obtained in the mass and density dependence of the extraction current, as well as the voltage at which transition from space-charge limited to ion saturation emission occurs.
\end{abstract}

KEY WORDS: Duopigatron; ion source; instability; source plasma; ion acoustic; sheath model; noble gas.

\section{INTRODUCTION}

In recent years a great deal of research has concerned the generation of dense ion source plasmas for high-current beam extraction. The duopigatron is an important ion source which has been extensively developed for neutral beam heating systems in plasma fusion experiments. A series of increasingly larger and more powerful duopigatron sources has been developed in the U.S. (at Oak Ridge National Laboratory ${ }^{(1-3)}$ ) and in Japan, ${ }^{(4)}$ to provide hydrogen and deuterium beams of up to tens of amperes at energies greater than $100 \mathrm{keV}$. Such sources have been used for heating many of the large magnetic confinement plasma devices.

'Department of Nuclear Engineering, The University of Michigan, Ann Arbor, Michigan 48109. 
In parallel with the development of these high-power sources, work has been performed in France ${ }^{(5-7)}$ and the Federal Republic of Germany ${ }^{(8)}$ to develop and study the duoplasmatron and small-area duopigatron as sources of both hydrogen and noble gas ions. Beam currents of tens of milliamperes at up to $30 \mathrm{kV}$ have been reported for duoplasmatron designs and a multiple-discharge duopigatron design.

The purpose of the experimental study reported here is to examine the performance of a large-area (ORNL type) duopigatron as a source of intermediate and high-mass ion beams including argon $(A=40)$, krypton $(A=84)$, and xenon $(A=131)$. There exist a number of important processing applications for large-current, high-mass beams including dry etching of semiconductors $^{(9)}$ and surface modification of materials. ${ }^{(10)}$ Other potential uses for such beams include plasma diagnostics, heavy-ion plasma heating, and preinjection into high-energy ion accelerators such as the RF quadrupole.

\section{EXPERIMENTAL CONFIGURATION}

The ion source used in the present study is a $10-\mathrm{cm}$ duopigatron of the type developed at Oak Ridge National Laboratory. Figure 1a depicts this duopigatron as configured for the present study. The discharge is initiated by thermionic emission from an oxide-coated, tungsten-wound, tantalum cathode. Feed gas enters the cathode region near the base of the filament. The formation of a double layer of electrons and ions between the cathode plasma and the anode (Fig. 1b) accelerates electrons into the anode volume, following the field lines of the source magnet. These primary electrons, with energies of $30-90 \mathrm{eV}$, ionize the gas in the anode region to form the source plasma. The first extraction grid is maintained at a negative potential with respect to the source plasma, by means of a $350-\Omega$ load resistor between the grid and second anode. The resulting potential profile reflects primary electrons, thus establishing a reflex ( $P$ enning $I$ on $G$ auge type) discharge between the double layer and the grid. This increases the path length and ionization efficiency of the primary electrons. Ions escape from the source plasma either by crossing the double layer toward the cathode plasma (necessary to maintain stability of the double layer) or by crossing the first grid sheath boundary to be accelerated by the extraction grids.

A triode extraction grid arrangement was used, with the second grid biased negatively at $10 \%$ of the primary accelerating voltage. The grids were approximately $52 \%$ transparent with 369 apertures of $3.75 \mathrm{~mm}$ diameter in a $10-\mathrm{cm}$-diameter hole pattern. 
a)

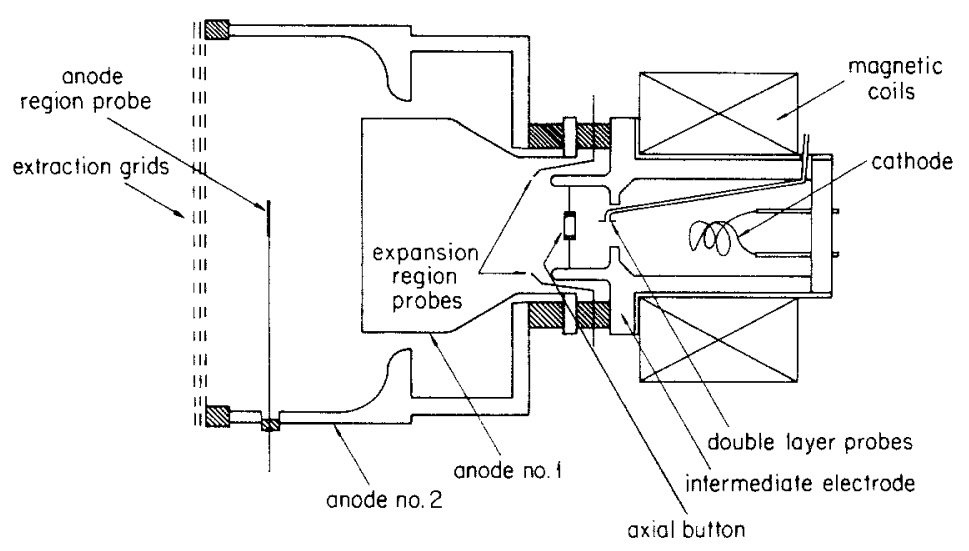

b)

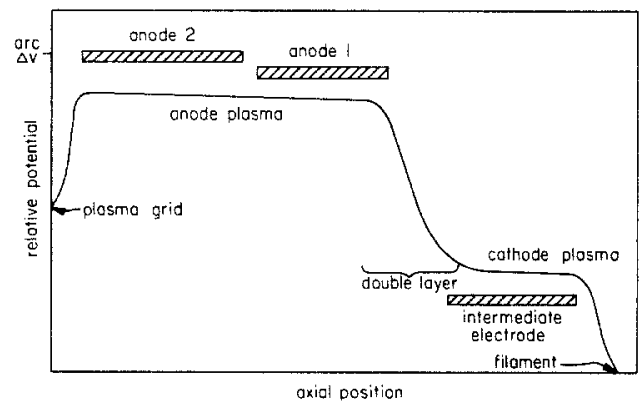

Fig. 1. (a) 10-cm diameter Duopigatron ion source, showing location of plasma probes; (b) potential profile through Duopigatron, showing double layer, and plasma grid sheath (see Ref. 3).

The entire experiment is shown schematically in Fig. 2. Downstream of the ion source is a 38 -cm-long, 30-cm-diameter neutralization tank in which a neutral gas background density is maintained by excess feed gas from the source. A gate valve, 15-cm-diameter drift tube, and target tank complete the beamline. Overall length from the extraction grids to the end of the target tank is approximately $163 \mathrm{~cm}$.

The high-voltage beam extraction circuit utilized an ignitron switched and crowbarred capacitive discharge system. A current-limiting resistor of $80 \Omega$ was used in series with the source, while a parallel resistor to ground was used to stabilize the current through the firing ignitron. 


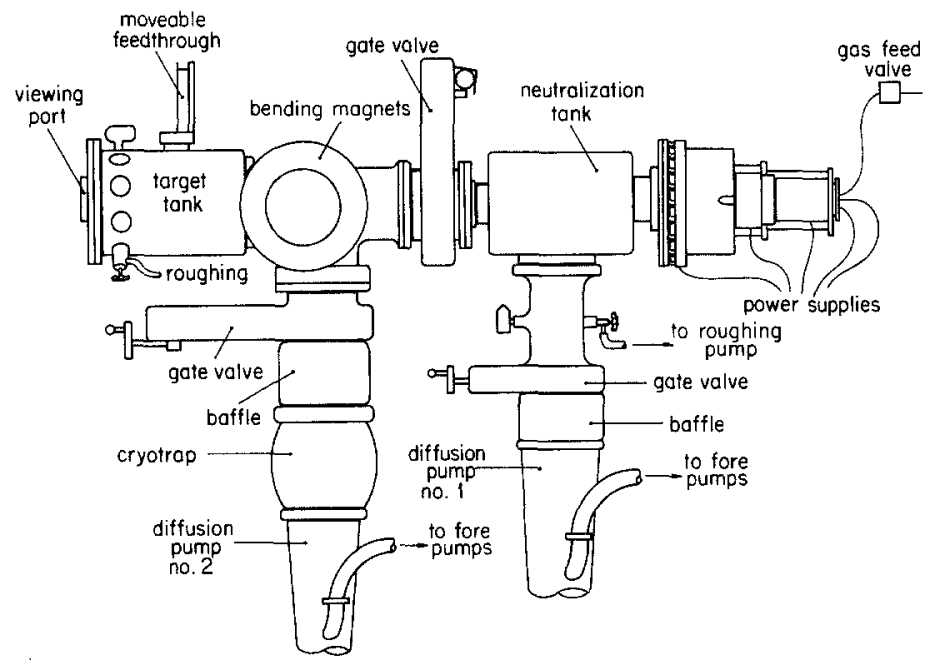

Fig. 2. Ion source and beamline experimental configuration.

\section{SOURCE PLASMA EXPERIMENTS}

For argon, krypton, and xenon, reproducible source plasma discharges were obtained with total arc currents up to approximately $40 \mathrm{~A}$ (limited by power supplies). In this study, arc current was controlled primarily by adjusting gas feed, rather than applied arc voltage. Lower gas feed rates and anode-region pressures were needed to sustain arcs in krypton and xenon than in argon. At an arc voltage of $100 \mathrm{~V}$ and a source magnet current of $7.1 \mathrm{~A}$ (corresponding to a peak magnetic field of $115 \mathrm{G}$ ), arc currents of approximately $22 \mathrm{~A}$ were obtained for argon at a gas feed rate of 0.14 Torrliter $/ \mathrm{sec}$ and a pressure of $3 \mathrm{mTorr}$, for krypton at $0.1 \mathrm{Torr}-\mathrm{liter} / \mathrm{sec}$ and $1 \mathrm{mTorr}$, and for xenon at 0.05 Torr-liter/ $\mathrm{sec}$ and $0.5 \mathrm{mTorr}$. This trend toward lower required pressures for the higher-mass gases can be explained to a large extent by observing that the ionization cross sections for $30-\mathrm{eV}$ electrons are approximately $2 \pi a_{0}^{2}, 3 \pi a_{0}^{2}$, and $4.3 \pi a_{0}^{2}$ for argon, krypton, and xenon, respectively ${ }^{(11)}$ (where $a_{0}$ is the first Bohr radius).

A source plasma instability has been observed for argon, krypton and xenon which is more pronounced than that observed for hydrogen under similar conditions. Because of the sensitivity of this instability to gas feed rate and its dual-mode character, it is believed to be an arc starvation instability of the type previously observed in studies of duoplasmatron sources by Lejeune ${ }^{12}$ and by Winter and Wolf. ${ }^{(13)}$ The instability is evidenced by a rapid shift between a "normal" (high-current) mode, and an "arc starvation" (low-current) mode. The arc-starvation mode is characterized 
by a low neutral gas density, with a resulting ionization density insufficient to provide the required ion flux to maintain a stable intermediate electrode double layer in accordance with the Langmuir ${ }^{(14)}$ sheath criterion $j_{e} / j_{i}=$ $\gamma\left(M_{i} / m_{e}\right)^{1 / 2}$, where $\gamma$ is a constant or order unity. In the experiments reported here, the parametric behavior of this instability has been studied.

To monitor the effects of this instability on the double-layer potential profile, a double probe was devised to measure within the double layer. The two probes, spaced $6 \mathrm{~mm}$ apart axially, did not necessarily bound the entire double layer, but did provide a relative measurement of the slope of the potential profile in this region.

Figures $3 \mathrm{a}$ and $4 \mathrm{a}$ show the current (lower traces) and double-layer potential difference (upper traces) for low-current-mode operation in argon and krypton, respectively. At a discharge current of approximately $1 \mathrm{~A}$, probe potential differences of 20 and $35 \mathrm{~V}$ are measured. Figures $3 \mathrm{~b}$ and $4 \mathrm{~b}$ show the high-current mode (during which current was ramped from approximately 3-12 A during 500-600 msec pulses). In this case, the probe potential difference is only $10 \mathrm{~V}$. Further, the overall arc voltage drops with increasing current. Thus, the high-current mode is characterized by a flatter and lower double-layer potential profile, and correspondingly lower primary-electron energy.

Figures $3 c$ and $4 c$ show the argon and krypton discharges during mode shifts between the low- and high-current cases. This mode shift can be explained by examining the behavior of the ionization cross sections in the range $30-100 \mathrm{eV}$. The cross sections peak at about $100 \mathrm{eV}$ and begin to drop rapidly below about $40 \mathrm{eV} .^{(11)}$ For the low-current discharge most of the arc voltage drop of 100-110 V occurs across the double layer, producing highenergy primary electrons with high ionization efficiency. The ions thus produced diffuse to the double layer to contribute to an increased ion flux toward the cathode. This permits (according to the sheath criterion) an increased electron flux and higher arc current. Now, however, the lowered double-layer accelerating potential produces lower-energy primary electrons with decreased ionization efficiency. A drop in ion production reduces the double-layer ion flux, forcing a drop in arc current. When the gas density is sufficient to sustain an adequate ion generation rate, with the lower-energy primary electrons, the mode shifting ceases and the arc enters a relatively stable high-current mode.

In this mode, however, the arc current displays a lower-amplitude sinusoidal oscillation as plotted in Fig. 5. It should be noted that these observed fluctuation frequencies (at $6 \mathrm{~A}$ magnet current) follow an ion acoustic mass scaling to within better than $10 \%$. Ion density fluctuations occur with negligible delay throughout the anode plasma volume, suggesting that these are transmitted by streaming electrons. It should also be noted 
a)

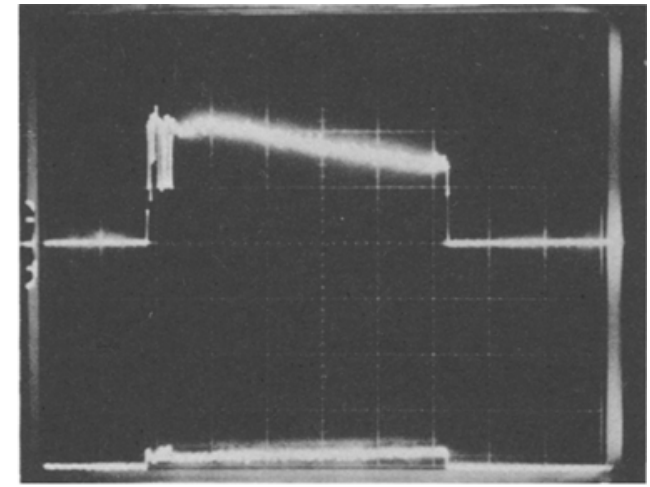

b)

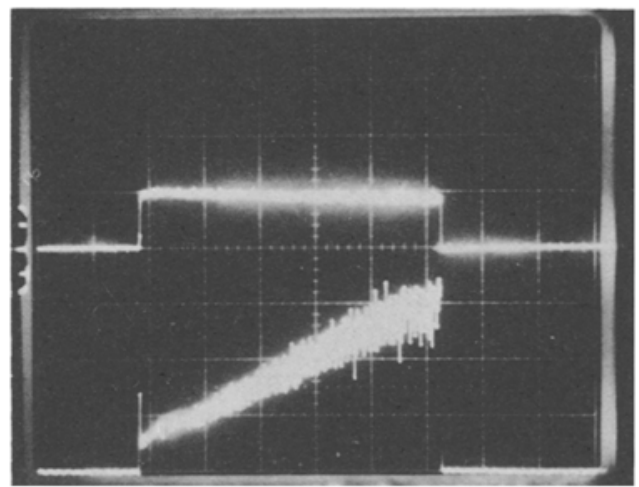

c)

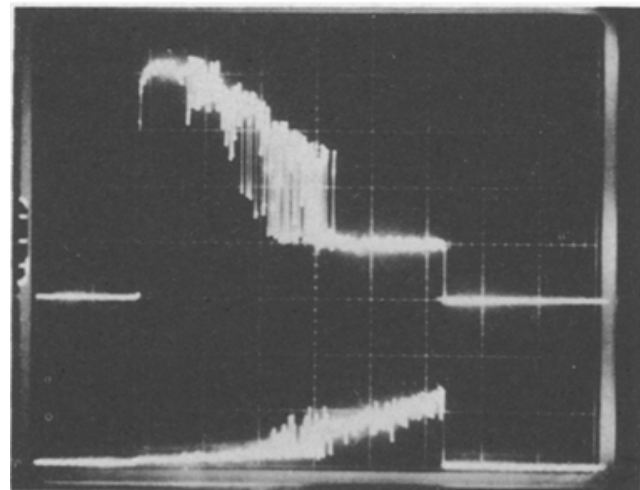

Fig. 3. Double-layer floating potential difference (upper trace, $10 \mathrm{~V} /$ div) and arc current (lower trace, $4 \mathrm{~A} /$ div) for argon in (a) low current mode, (b) high current mode, and (c) mode-shifting condition. Time scale is $100 \mathrm{msec} /$ div. 
a)

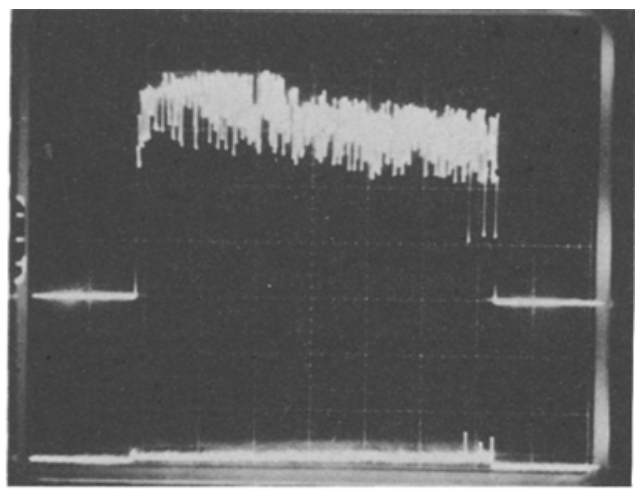

b)

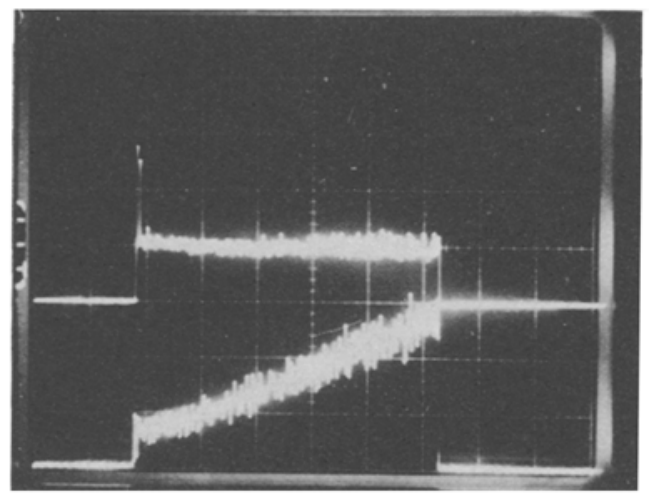

c)

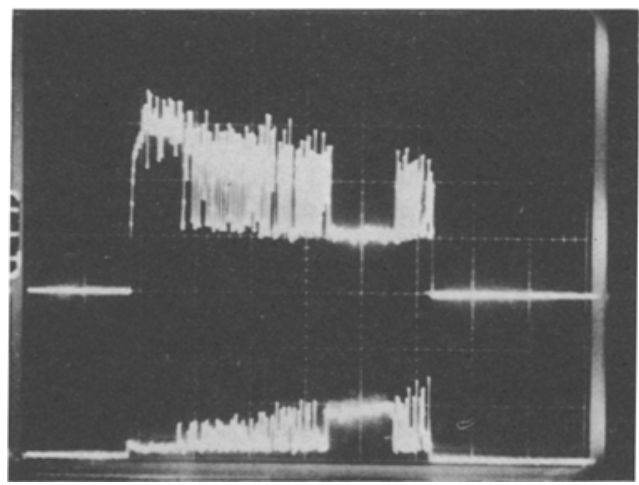

Fig. 4. Double-layer floating potential difference (upper trace, $10 \mathrm{~V} / \mathrm{div}$ ) and arc current (lower trace, $4 \mathrm{~A} / \mathrm{div}$ ) for krypton in (a) low current mode, (b) high current mode, and (c) modeshifting condition. Time scale is $100 \mathrm{msec} /$ div. 


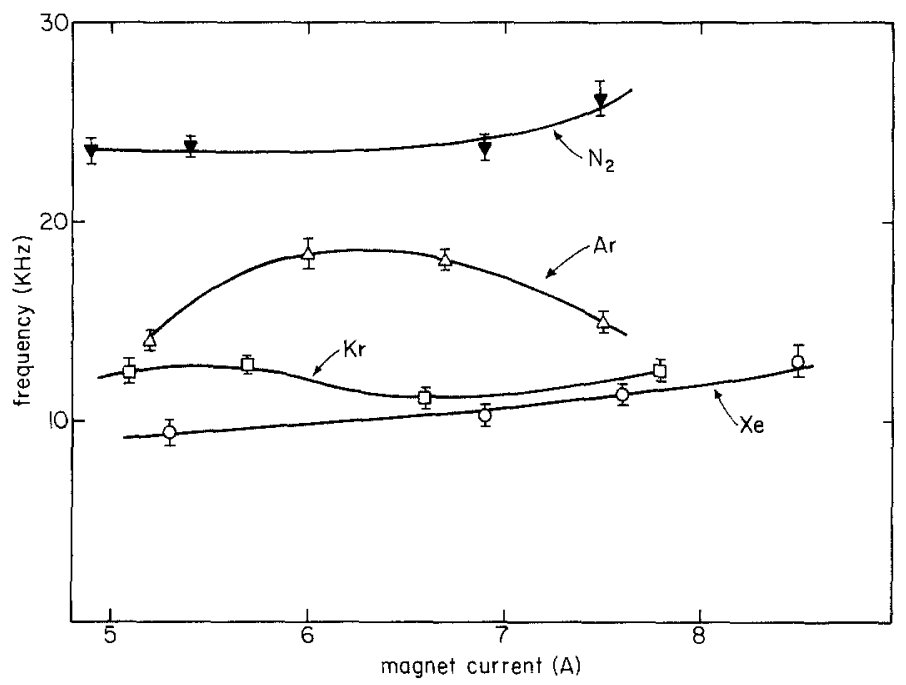

Fig. 5. Arc oscillation frequency as a function of source magnet current.

that the insensitivity of the oscillation frequency to magnetic field rules out any EXB rotational oscillation as the cause.

Unlike the duoplasmatron used in the studies cited above, ${ }^{(12,13)}$ the duopigatron is characterized by a reflexing electron discharge in the anode region. To determine the effect of these electrons on the mode shift instability, discharges were run with and without the load resistor between the plasma grid and the anodes. Without the resistor (grid shorted to anodes) the reflexing electrons will be suppressed. In this case, the discharge remains in the low-current mode for a longer period, and becomes destabilized in the high-current mode. Two possible mechanisms may be responsible for this behavior. The reflexing electrons normally help to neutralize the space charge on the anode side of the double layer. Without these electrons the potential profile could be steepened, and the double layer would resist the transition to the smoother potential profile characteristic of the high-current mode. Also, the reflexing electrons contribute to the ionization density, and their absence would have the same effect as a lowered gas density. Probe measurements of the anode-region ion density and the potential slope in the double-layer region indicated a slight decrease in ion density without the reflexing electrons, but no measurable change in the double layer near the snout. The reflexing electrons apparently influence the mode shift instability through their effect on ionization density, rather than space charge. 

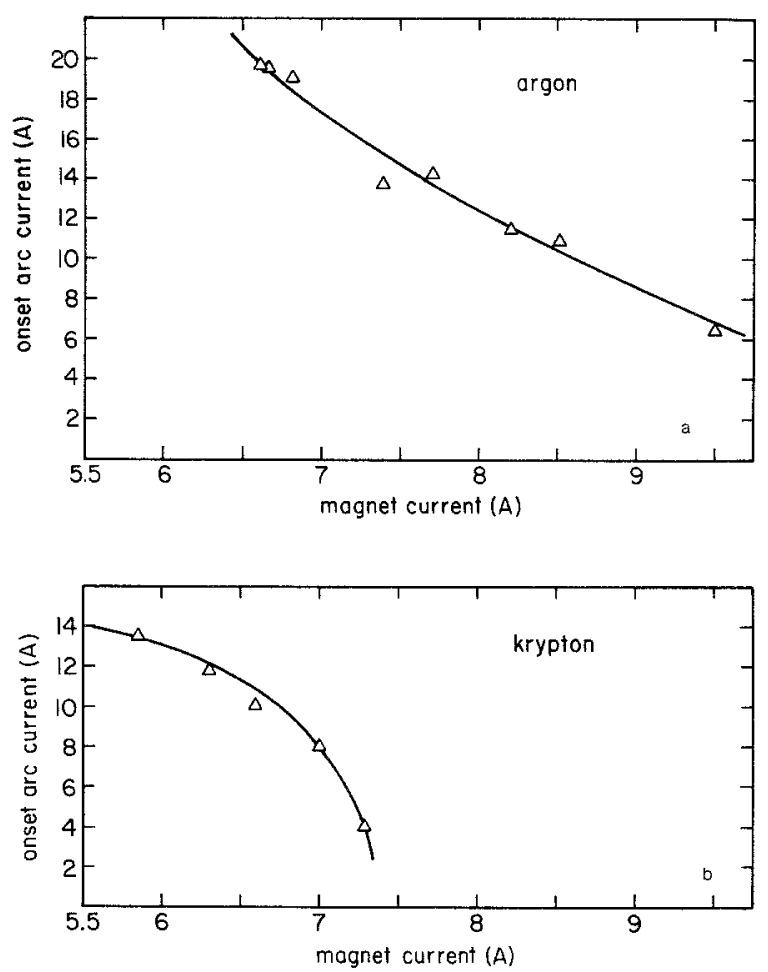

Fig. 6. Mode shift onset current as a function of source magnet current for (a) argon and (b) krypton.

Figures $6 \mathrm{a}$ and $\mathrm{b}$ show the mode-shift onset current as a function of source magnet current for argon and krypton. At higher field strengths a lower total arc current is required to initiate the instability. Studies by Jacobsen and Eubank ${ }^{(15)}$ have shown that the double-layer potential difference is proportional to the temperature of the double-layer electrons. Electron loss will be controlled by Bohm diffusion where $D_{\perp \text { Bohm }} \propto T / B$. Thus for a given steady-state density condition, with constant electron generation and loss, the double-layer electron temperature (and potential difference) will be proportional to the magnetic field strength. As the field strength is increased, the double-layer voltage difference increases. Thus (for a fixed power-supply voltage), the maximum current attainable, before forcing a change in the double-layer configuration to the high-current mode, will decrease. The difference in instability onset current between argon and krypton is believed to be due to a difference in arc power-supply voltage. 


\section{BEAM EXTRACTION}

\subsection{Review of Extraction Theory}

The ion current which can be extracted from a source plasma is limited by the density and mobility of the source ions. The theory developed by Lejeune $^{(16)}$ to predict saturation ion extraction currents utilizes a sheath boundary model to determine the potential profile and ion density near a biased electrode. The region between the bulk source plasma and the electrode (in this case an extraction grid) is considered to consist of two regions; a presheath region in which $n_{e}=n_{i}$, but $E \neq 0$, and the sheath region in which $n_{e}<n_{i}$ and $E \neq 0$. The axial energy of ions crossing from the presheath to the sheath regions is found to $b \mathrm{e}^{(17)}$

$$
W_{\mathrm{i}}=\frac{1}{2} k T_{\mathrm{e}}
$$

By assuming the ion energy in the bulk plasma region to be small relative to the electron energy, the potential difference across the presheath is found to be

$$
V_{\mathrm{ps}}=k T_{\mathrm{e}} / 2 e
$$

Thus the electron (and ion) density at the sheath-presheath boundary is

$$
n_{\mathrm{es}}=n_{\mathrm{is}}=n_{\mathrm{e} 0} \exp \left(-e V_{\mathrm{ps}} / k T_{\mathrm{e}}\right)
$$

Substituting for $V_{\mathrm{ps}}$ we find

$$
n_{\mathrm{is}}=0.61 n_{\mathrm{e} 0}
$$

The ion saturation current then becomes ${ }^{(16)}$

$$
j_{\mathrm{i}}=0.61 n_{\mathrm{e} 0} \mathrm{e}\left(k T_{\mathrm{e}} / M_{\mathrm{i}}\right)^{1 / 2}
$$

This derivation assumed a Maxwellian distribution of electron velocities in the presheath region. However, the duopigatron is characterized by a large number of primary electrons reflexing between the plasma grid and the double layer at the intermediate electrode. These electrons, which determine the potential of the plasma grid, will enter the presheath region with energies of approximately $30-90 \mathrm{eV}$ (compared to a bulk electron temperature of $1-2 \mathrm{eV}$ ). To more accurately characterize the sheath and the resulting ion saturation current, modifications to the Bohm theory have been proposed by Prewett and Allen ${ }^{(18)}$ and Uehara et al ${ }^{(19)}$ These theories include the effects of a second, higher-energy, group of electrons in the Poisson equation. According to Prewett and Allen, the boundary between the sheath and presheath is characterized by a potential $V_{0}$, where

$$
e V_{0} / k T_{\mathrm{c}}=\frac{\frac{1}{2}\left(n_{\mathrm{i} 0} / n_{\mathrm{e} 0}\right)}{\left(1-j_{\mathrm{b}} / j_{\mathrm{c}}\right)}
$$


where $j_{\mathrm{b}}$ is the current density of beam electrons and

$$
j_{\mathrm{c}}=n_{\mathrm{e} 0} \mathrm{e}\left(k T_{\mathrm{e}} / m_{\mathrm{e}}\right)^{1 / 2}\left(2 e V_{0} / k T_{\mathrm{e}}\right)^{3 / 2}
$$

Note that for $n_{\mathrm{e} 0}=n_{\mathrm{i} 0}$ and $j_{\mathrm{b}}=0$ this model reduces to the Bohm prediction.

Typical parameters of this experiment have been measured by Langmuir probe:

$$
\begin{gathered}
j_{\mathrm{b}}=0.5 \mathrm{~A} / \mathrm{cm}^{2} \\
k T_{\mathrm{e}}=2 \mathrm{eV} \\
n_{\mathrm{e} 0}=n_{\mathrm{i} 0}=1 \times 10^{11} \mathrm{~cm}^{-3}
\end{gathered}
$$

Solving Eqs. (6) and (7) iteratively we find

$$
V_{0}=0.72 k T_{\mathrm{e}} / e
$$

Then we obtain the ion density from

$$
n_{\mathrm{i}}=n_{\mathrm{e} 0} \exp \left(-e V_{0} / k T_{\mathrm{e}}\right)=0.49 n_{\mathrm{e} 0}
$$

and the ion velocity becomes

$$
\frac{1}{2} M_{\mathrm{i}} v_{\mathrm{i}}^{2}=e V_{0}
$$

Substituting for $V_{0}$ from Eq. (8) we have

$$
v_{\mathrm{i}}=\left(1.44 k T_{\mathrm{e}} / M_{\mathrm{i}}\right)^{1 / 2}
$$

The ion current can then be written

$$
j_{\mathrm{i}}=0.58 n_{\mathrm{e} 0} \mathrm{e}\left(k T_{\mathrm{e}} / M_{\mathrm{i}}\right)^{1 / 2}
$$

Thus, for the parameters of this experiment, the ion saturation current reaching the plasma grid should not vary significantly from the Bohm criterion prediction of Eq. (5).

\subsection{Beam Extraction Experiments}

Argon, krypton, and xenon ion beams have been generated at varying extraction voltages and source plasma densities to determine the scaling of extracted current with these parameters (Figs. 7a, b, and c). For argon, the high-voltage drain current appears to approach a plateau for each arc current value, as predicted by the Lejeune theory. However, for krypton and particularly xenon, drain current does not saturate for the high arc current cases. The argon drain current scales approximately as $I_{\text {arc }}^{5 / 4}$. Since ion density scales approximately as the arc current, and electron temperature increases slightly with higher current, this scaling is reasonable and in good agreement with extraction theories [Eq. (5)]. It appears, however, that for the higher- 

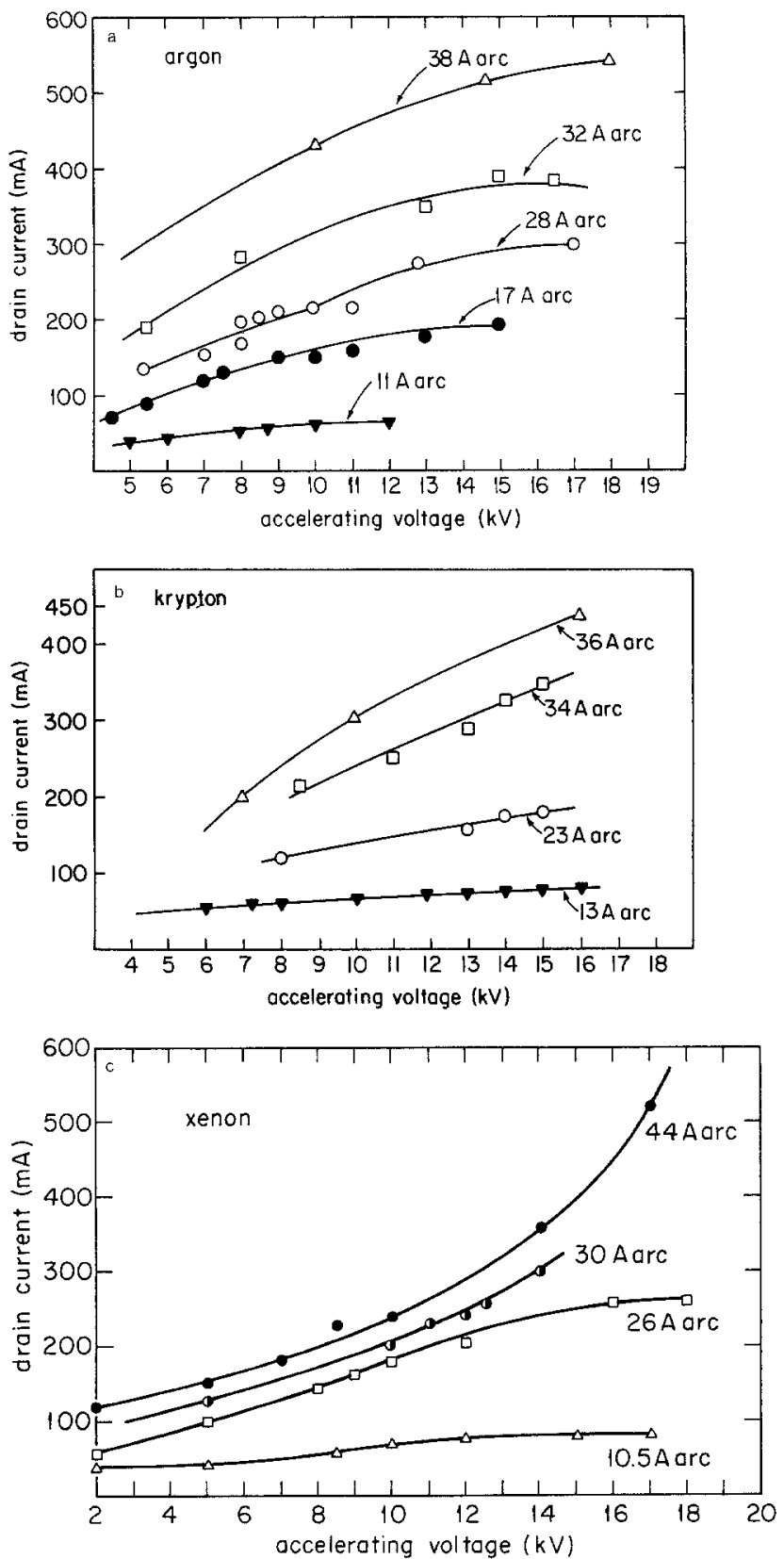

Fig. 7. High-voltage drain current as function of arc current and accelerating voltage for (a) argon, (b) krypton, and (c) xenon. 
mass ions a somewhat different dependence is involved. Lejeune ${ }^{(16)}$ provides a prediction of the accelerating voltage at which the extracted current should undergo the transition from space-charge limited emission to the ion saturation regime:

$$
V^{*}=\left[9 j_{+} d^{2} / 4 \varepsilon_{0}(2 e)^{1 / 2}\right]^{2 / 3} M^{1 / 3}
$$

where $j_{+}$is the ion current density of Eq. (5) and $d$ is the grid spacing $(14 \mathrm{~mm})$. This equation gives the voltage at which the space-charge limited (Child-Langmuir) current equals the ion saturation current of Eq. (12). Applying this prediction to the experimental case, for a drain current of $400 \mathrm{~mA}, V^{*}$ is $18 \mathrm{kV}$ for argon, $23 \mathrm{kV}$ for krypton, and $27 \mathrm{kV}$ for xenon. At $160 \mathrm{~mA}$ drain current, $V^{*}$ is $11 \mathrm{kV}$ for argon, $14 \mathrm{kV}$ for krypton, and $15 \mathrm{kV}$ for xenon (see Figs. 7a, b, and c). Thus, for argon we operated in the region of $V \geqslant V^{*}$; for krypton, in general, $V \leq V^{*}$; and for xenon $V<V^{*}$.

For xenon, at the higher arc currents, the extraction current is apparently not limited by ion density and mobility, as the sheath extraction theory assumes, but rather by normal space-charge limited emission. The xenon extraction currents, for the dense-source plasmas, in the range 10$17 \mathrm{kV}$, follow a $I \propto V^{3 / 2}$ dependence as predicted by the Child-Langmuir law for space-charge limited emission at $V \ll V^{*}$. In this regime, the ion density is well above that required to provide the measured extraction current, and thus the source plasma is acting as an emissive surface with an excess supply of ions.

At the other extreme, the argon extraction closely follows the Lejeune sheath model extraction theory, both in terms of extraction current scaling with ion density, and in its approach to saturation with increasing acceleration voltage. Between these extremes, the krypton-extracted currents follow an almost linear dependence indicating a transition from space-charge limited current to a saturable current as $V$ approaches $V^{*}$.

For the argon extraction currents, which appear to follow the sheath extraction theory, excellent quantitative agreement exists between theory and experiment. For example, at an arc current of $32 \mathrm{~A}$, Langmuir probe measurements indicate a peak plasma density of $6 \times 10^{11} \mathrm{~cm}^{-3}$, a radial average density of $4.9 \times 10^{11} \mathrm{~cm}^{-3}$, and an electron temperature of approximately $2 \mathrm{eV}$. Equation (12) would then predict an average ion saturation current density reaching the plasma grid of $9.9 \times 10^{-3} \mathrm{~A} / \mathrm{cm}^{2}$. Since the grid apertures cover $79 \mathrm{~cm}^{2}$ with a transparency of $52 \%$, the saturation extraction current should be $407 \mathrm{~mA}$. This in excellent agreement with the experimentally determined value of $390 \mathrm{~mA}$. Further, from Eq. (13), saturation should occur at approximately $17 \mathrm{kV}$, which is also supported by the data. 


\section{ACKNOWLEDGMENTS}

We acknowledge the assistance, in the form of equipment and advice, of the Plasma Technology group of the Fusion Energy Division of Oak Ridge National Laboratory, especially W. L. Stirling and H. H. Haselton. This research was supported by the Air Force Office of Scientific Research, Contract 80-0029. P. D. Weber is supported under appointment to the Magnetic Fusion Energy Technology Fellowship program which is administered for the U.S. Department of Energy by Oak Ridge Associated Universities.

\section{REFERENCES}

1. R. C. Davis, T. C. Jernigan, O. B. Morgan, D. L. Stewart, and W. L. Stirling, Rev. Sci. Instrum. 46, 576 (1975).

2. W. L. Stirling, C. C. Tsai, and P. M. Ryan, Rev. Sci. Instrum. 48, 533 (1977).

3. C. C. Tsai, W. L. Stirling, and P. M. Ryan, Rev. Sci. Instrum. 48, 651 (1977).

4. H. Horiike, M. Akiba, Y. Arakawa, S. Matsuda, and J. Sakuraba, Rev. Sci. Instrum 52, 567 (1980).

5. C. Lejeune, Nucl. Instrum. Methods 16, 429 (1974).

6. C. Lejeune, J. P. Grandchamp, J. P. Gilles, and J. Aubert, IEEE Trans. Nucl. Sci. 23, 1084 (1976).

7. C. Lejeune, J. P. Grandchamp, and J. Aubert, Rev. Phys. Appl. 12, 1835 (1977).

8. R. Keller and H. Winter, Part. Accel. 7, 77 (1976).

9. O. Okada, private communication, Hitachi Corp.

10. M. D. Nahemow, Conference Record of the IEEE Int. Conf. on Plasma Science, San Diego, California, 1983, IEEE Cat. No. 83CH1847-3.

11. S. C. Brown, in Basic Data of Plasma Physics, M.I.T. Press, Cambridge (1967), p. 143.

12. C. Lejeune, Ph.D. thesis, Orsay, France (1971).

13. H. Winter and B. H. Wolf, Plasma Phys. 16, 791 (1974).

14. I. Langmuir, Phys. Rev. 33, 954 (1929).

15. R. A. Jacobsen and H. P. Eubank, Plasma Phys. 15, 243 (1973).

16. C. Lejeune, in Applied Charged Particle Optics, A. Septier, ed., Academic Press, New York (1983), pp. 207-227.

17. D. Bohm, in Electric Discharges in Magnetic Fields, A. Guthrie and R. K. Wakerling, eds., McGraw-Hill, New York (1949), pp. 77-86.

18. P. D. Prewett and J. D. Allen, Proc. Phys. Soc. London, Sec. A 348, 435 (1976).

19. K. Uehara, K. Yatsu, S. Hagiwara, and S. Kojima, Jpn. J. Appl. Phys. 14, $176 \mathrm{l}$ (1975). 